\title{
The Language Reality and Strategies to Be Taken for the Standardization of Yi Character
}

\author{
Haixia Jia \\ Southwest Institute of Nationalities, Southwest Minzu University, Chengdu, China \\ Email: 317187051@qq.com
}

How to cite this paper: Jia, H. X. (2019). The Language Reality and Strategies to Be Taken for the Standardization of Yi Character. Open Journal of Modern Linguistics, 9, 551-560.

https://doi.org/10.4236/ojml.2019.96040

Received: November 21, 2019

Accepted: December 15, 2019

Published: December 18, 2019

Copyright $\odot 2019$ by author(s) and Scientific Research Publishing Inc. This work is licensed under the Creative Commons Attribution International License (CC BY 4.0).

http://creativecommons.org/licenses/by/4.0/

\begin{abstract}
This paper combs the current development status of Yi character in Sichuan, Yunnan, Guizhou and Guangxi, combined with the needs of social development, the approval of the National Yi language Terminology Standardization Working Committee, which be reported to the higher authorities for the implementation of the "General Yi Language Standard Scheme", and its implement effective. It explores the realistic basis and path choice of the proverbs.
\end{abstract}

\section{Keywords}

Standardization of Yi Character, Promote Trial, Countermeasure and Suggestion

\section{Introduction}

Language norms have been an important part of Chinese language policy and planning since ancient times. As early as the late Warring States period, ancient sages sprouted language norms. Xunzi was the first master of the Chinese preQin elaboration of language normative thoughts. He proposed two principles of language norms: First, "traveling the old" and "making new" simultaneously; second, the proper name from the old, the scattered name from afar, far from the middle. Qin Shihuang's "Books with the Same Text” policy, Tang Taizong's normative interpretation of the scriptures, Ming Taizu's "Abandon Mongolia to restore China" language reform, and the establishment of various ethnic minority regimes (such as Liao, Xixia, Jin, Yuan, Qing), The national script was created and promoted as "national language", "national character" and "writing system" (Zhou, 2017: pp. 74-80). It can be seen that language norms are a historical category, which develops with the development of society. The object, purpose and task of language specification will be different in different historical periods. 
After the founding of New China, the party and the state attached great importance to the standardization of national languages. Since the 1950s, the predecessors of Chinese linguistics began to record Chinese dialects and minority languages using modern linguistic survey methods. During this period, the standardization of language among ethnic groups was also one of the important contents.

The $\mathrm{Yi}$ is one of China's countries with a long history and ancient culture. According to the data of the sixth census in 2010, the Yi is the sixth largest ethnic minority after Zhuang, Hui, Manchu, Uyghur and Miao, with a total population of $87,143,933$. It is mainly distributed in the northwestern parts of Sichuan, Yunnan, Guizhou and Guangxi Zhuang Autonomous Region. Liangshan Yi Autonomous Prefecture in Sichuan province is the largest compact community, with a population of 2,226,755. Other areas are mostly mixed areas.

The Yi have their own language and characters. The Yi language belongs to the Tibetan-Burmese language branch of the Sino-Tibetan language family. It is divided into six dialects: the Eastern dialect, the southeast dialect, the southern dialect, the western dialect, the central dialect and the northern dialect. Yi character is originally a hieroglyph. There are many words in the ancient literatures of Sichuan, Yunnan, and Guizhou that are pictographs. Since the Qin and Han Dynasties, the Yi character has been in the transitional stage from the ideographic to the phonetic, that is, the combination of ideology and phonetic. Among them, there are more ideograms in Yunnan and Guizhou, and there are more vocal compositions in Sichuan, which is in line with the development of characters from pictographic to ideographic, from ideographic to fidelity. Since the modern times, the Yi character in Yunnan and Guizhou has maintained the characteristics of ancient features, while the Sichuan Yi character has a great development (Ma, 2012: p. 14).

\section{Realistic Foundation}

Yi character is a symbol for recording proverbs. It is an ancient and distinctive text that records tens of thousands of classical Yi literature. The creation, standardization and reform of Yi character are the most important links in the work of the Chinese language. They directly affect the work of the Yi cultural heritage, basic education, publishing and printing, information processing and social and economic development, as well as domestic and international. The efficiency of language lovers to learn and use.

\subsection{Yi Character Is of the Same Origin in the Four Provinces}

Judging from the existing research data, most scholars believe that the essays in the three provinces of Yunnan, Guizhou, Sichuan, and Guangxi should originate from the same mother tongue, and each develops due to factors such as times and regions. The legends of the literary creations that have been circulated throughout the country are only records of the collation and standardization 
work used by local essays. To this day, we can still find the connection between the three provinces and one district in the comparative study of the forms and meanings of the texts.

The differences between the localities are mainly reflected in the writing style and habits. Generally speaking, the Sichuan Yi character is in the shape of a rectangular erect, and the Yunnan and Guizhou Yi characters are flat and horizontal. If the Sichuan essay is standing, then the Yunnan and Guizhou Yi character are horizontal, the former is thin and tall, and the latter two are flat.

\subsection{The Needs of Society}

In the mid-1990s, government functions at all levels considered the immediate interests and actual needs of ethnic minorities too much, and adopted various measures to promote the popularization of Chinese language. It is a good thing to look at the society. However, if the emotional and cognitive factors of ethnic minorities are not fully considered and the long-term needs of ethnic minorities for the inheritance of traditional cultures are neglected, additional problems will arise. Once the economic development of ethnic minority areas has been solved and their food and clothing has been solved, their national consciousness will be strengthened, and there will be more spiritual needs. They will consider cultural issues, ethnic identity issues, and the development of their own spoken and written languages. This is indeed the case. In recent years, with the support of relevant national policies, the economy of the Yi nationality has flourished and various undertakings have achieved remarkable results. The Yi people have begun to pay attention to and appeal to the inheritance and protection of the traditional culture of the nation, and have begun to practice from various fields (Wang, 2007).

\subsubsection{The Policy Level}

Local language work offices have issued special documents to guide the use of national language and characters, such as "Regulations on the work of the languages of ethnic minorities in Yunnan Province (2013)" "Regulations on the language of the Yi people in the Liangshan Yi Autonomous Prefeture (2009)". These regulations aim to clearly define the fields, occasions, norms and other content used in national languages.

\subsubsection{The Education Level}

Southwest Minzu University set up a master's degree and doctoral degree in 2013. This is also the first institution in China that combines undergraduate and postgraduate education. It is also equipped with the Yi Museum and the Yi Literature Library as a teaching practice base. Two graduates have written their dissertations with Yi characters. The Sichuan Provincial Department of Education officially issued the "Notice on Actively Promoting the Continuous and Coordinated Development of Bilingual Education" in 2017, stating that Sichuan Province will begin its college entrance examination in 2020. All types of candidates 
except Chinese, English, and all subjects will be tested in Yi characters. The second-class mode candidate, Yi character is listed as one of the test subjects, time 120 points, a total score of 150 points.

The Chuxiong Teachers College in Yunnan Province began to enroll in the Department of Language and Literature in 2019, and cultivated the backbone of Yi language teachers for primary and secondary schools.

\subsubsection{The Employment Level}

Under the baton of the state's advocacy of "scientific protection of the language and language of all ethnic groups" ${ }^{\prime}$, including all walks of life primary and secondary schools, universities, civil servants, etc. have set up special posts to recruit graduates who are proficient in national language and writing. It has stimulated the enthusiasm of students to learn Yi language and character, and changed "I want to learn" to "I want to learn." In particular, the recognition and enthusiasm of the students of their own countries for their mother tongue has risen.

\subsection{The Development of Yi Language in Four Provinces}

According to the laws governing the development of language and characters, since the 1950s, relevant departments and experts in Sichuan, Yunnan, Guizhou, and Guangxi have successively adopted the normative norms as the starting point, starting from the long-term interests of enriching and developing the Yi language and culture. The national language policy of "all ethnic groups have the freedom to use and develop their own spoken and written languages", collating and standardizing the traditional essays in their respective jurisdictions, and experimenting in various areas of the Yi nationality, and have achieved different levels of practical effects, especially in 1980. The "Yi language standard program" approved by the State Council played an important role in promoting the development of various social undertakings in Liangshan.

\subsubsection{Yi Character of Sichuan}

Sichuan is the largest inhabited area of the Yi nationality. The standardization work of the Yi language started earlier and the experience was richer. In the 1950s, the Fourth Task Force of the Minority Language Survey of the Chinese Academy of Sciences conducted an in-depth investigation into the use of Yi language in Liangshan, Sichuan, and designed "The Xikang Yi New Text Experiment Program" "the Liangshan Yi People's Pinyin Text Scheme", and "Yi language standard program". After many years of practice, the Liangshan people finally chose the "Yi language standard program", which was officially approved by the State Council in 1980 in the Yi area of Sichuan Province.

${ }^{1}$ In October 2011, the decision of the Central Committee of the Communist Party of China on the major issues of deepening the reform of the cultural system to promote the great development and prosperity of socialist culture adopted by the Sixth Plenary Session of the 17th Central Committee of the Communist Party of China put forward the decision of "scientific protection of the language and characters of all ethnic groups". 


\subsubsection{Yi Character of Yunnan and Guizhou}

The distribution characteristics of the Yi people in Yunnan and Guizhou are mainly small settlements and large dispersions.

In 1982, the National Committee for the Work of Ethnic Languages and Languages of Yunnan Province began to explore and study the issue of the standardization of the Yi language, and proposed to design a set of ideographic and essay schemes applicable to all parties based on the original local essay. In July 1983, it developed and determined 1675 common ideograms in the first batch of standard texts. After three years of trials, 580 additional texts were added, and 350 loan words were written, resulting in a total of 2258 words, basically solving the problem of expressing modern proverbs and new terminology. However, due to many dialects, large differences, and many words, the program was stopped in 1989.

The work of the Yi language in Guizhou Province was gradually developed from the translation, collation and regulation of the ancient books in the province. In 1955, with the attention and support of relevant departments, the Ethnic Affairs Commission of Bijie Prefecture of Guizhou Province established the Yi Character Translation Group to organize, translate and publish a large number of books on history. In the 1960s, the institution was revoked, and the translation and organization of the ancient books of the Yi people were also interrupted until 1977. In 1980, according to the wishes of the masses, the provincial party committee carried out the pilot work of bilingual teaching in the province, mainly focusing on common words and daily conversations, but did not explicitly mention the problem of standardization.

\subsubsection{Yi Character of Guangxi}

The ancient Yi language of Guangxi is of the same origin as that of Yunnan, Guizhou and Sichuan (Yang, 2002: pp. 378-387). In 1980, according to the spirit of the National Yi character Work Conference, Longlin County established the $\mathrm{Yu}$ character rescue team to collect and organize the ancient texts of the county. At the same time, it also sent a special person to the Yi language Translation Group of Bijie City, Guizhou Province to study, and co-edited "Longlin Yi People's Writings" (Mimeo), which is nearly 500 interpretations for ancient Yi character. At present, there are only 10 volumes of relatively complete essays circulating in the private collections in Guangxi, about 100,000 words, but no one can read them completely, and there are no people who specialize in ancient literary studies.

\subsection{The Present Situation and Characteristics of the Development of Yi Language in China}

When developing and standardizing the original essays in the provinces and regions, the localities discussed the problems of the four provinces and regions from different angles, and also put forward many ideas. However, no comprehensive statistics has been made on the number of words in Yi characters. From 
the various printed materials, Sichuan Liangshan has "Yi Language Word Table" (more than 800 words); Guizhou has "Yi Language Dictionary" (more than 6500 words); Shuangbai in Yunnan has "Yi Language Dictionary" (more than 2000 words); Shi Lin in Yunnan has "Yi-Chinese Concise Dictionary" (1050 words).

In 1998, the Leading Group for the Arrangement and Publication of Yi Characters Ancient Books and Yi societies of Yunnan, Guizhou and Sichuan provinces jointly proposed a plan to create a unified text, and established a unified writing office in Yunnan. During the period, the writing team collected all the essays in the country and standardized more than 2800 Yi characters. After more than two years of training practice, due to the lack of mass foundation, the study was suspended in 2002.

In October 2004, the tenth collaboration meeting of the Arrangement and Publication of Yi Characters Ancient Books made a summary of the "Regulations on the Standardization of the Yi Language" approved by the State Council. The minutes pointed out that since the founding of the People's Republic of China, especially since the State Council approved the implementation of the standard essay in 1980, the work of the essay has been rapidly developed and has achieved gratifying results. The practice in the past 30 years proves that the normative text is in line with reality and practical, and has made positive contributions to the social and economic development of the Yi area and to promoting exchanges between ethnic groups.

In the next few years, relevant experts and institutions worked together to comprehensively launch the development and trial work of the "General Yi Language Standard Scheme", and also published related textbooks and reference books. In November 2014, the fifteenth collaboration meeting of the Arrangement and Publication of Yi Characters Ancient Books and the Tenth National Yi studies academic seminar were held in Xichang, Liangshan Prefecture. The General Assembly reviewed the "general Yi language standard scheme". From the formulation to the training and promotion for three years, the "General Yi Language Standard Scheme" has repeatedly sought opinions and has been continuously revised and improved. It has been recognized and supported by people from all walks of life in Yunnan, Guizhou, Sichuan and Guangxi. And decided to officially promote the use of the four provinces.

\section{Trial Effect of the "General Yi Language Standard Scheme"}

Since its trial in 2012, the "General Yi Language Standard Scheme" has achieved gratifying results (Jia \& Sha, 2014: pp. 19-24). There are Southwest Minzu University, National Civil Affairs and Humanities and Social Sciences Key Research Base-Yi Research Center of China, National Yi language Terminology Standardization Working Committee, Committee for the Compilation and Approval of Yi-Chinese Bilingual Textbooks in Colleges and Universities, and National Civil Affairs Committee Bilingual Talent Training Base and other units. Under the organization and coordination of the above unit, the work is carried out in various forms through various channels, mainly in the following aspects (Jia \& 
Sha, 2015: pp. 45-50):

1) Conduct a series of theme training courses

The National People's Committee Bilingual Talent Training Base has successfully launched 11 sessions of the "General Yi Language Standard Scheme" in Yunnan Honghe, Zhaotong, Chuxiong Prefecture, Sichuan Chengdu, Xichang, and Guizhou Guiyang, Bijie and Liupanshui. A total of more than 1260 trainees. The main components of the students are: the staff of the civil affairs commissions and the compiling departments at all levels in the four provinces, the teaching staff and workers of the colleges and universities, the teachers of the local primary and secondary schools (one type of model), and the national publishing workers. The training time is generally 7 to 10 days, and the passing rate of the students is $100 \%$. The students generally reflect that the general Yi character is easy to learn and use.

2) Cultivate high-level Yi language and character workers

Southwest Minzu University has established he only Yi nationality learning program in China. In 2013, it began to recruit masters and doctoral students. During the admissions examination, students' individual language listening, speaking, reading and writing skills are examined separately. After graduation, most of the students returned to the Yi nationality to engage in primary and secondary education, which made the relevant policies on ethnic language work in various places smoothly. In particular, the Sichuan Provincial Department of Education issued a notice in 2017, demanding that the 2020 college entrance examination increase the policy of Yi language testing, which inspires the enthusiasm of the Yi people to learn the general norms.

Yunnan Chuxiong Normal College opened its undergraduate major in linguistics and literature in 2019, and enrolled 50 Yunnan students who belonged to the six major dialect areas of Yi language. The courses offered were based on general norms and ancient literature.

3) Edit and publish all kinds of books at all levels

In order to cooperate with the training class, we have now compiled "The Guide of Yi Language's Common Nouns Chinese-Yi contrast” “General Standard of Yi Text" "Learn Yi language With me" "Basic Yi Language" "General Specification Yi Language Dictionary" "Illustrated Dictionary of Yi Animals" "Dictionary of Common Terms in Yi Language" and other related texts (including electronic version). In addition, local Yi language working organization have also taken corresponding measures to vigorously support the promotion and popularization of the "General Yi Language Standard Scheme".

The Language Committee in Guizhou Provincial has clearly stated that the preparation of primary school language teaching materials requires the use of general Yi pinyin for phonetic transcription. Six volumes have been prepared, of which the first, second and third volumes have been published and put into use, and the effect is good.

The Committee for the Compilation and Approval of Yi-Chinese Bilingual 
Textbooks in Colleges and Universities has compiled the "Southwest Minzu University Ancient Books Library" in the form of the three lines of ancient Yi characters, the common Yi language pinyin, and the Chinese translation. The series consists of 10 volumes, including 3 volumes of Guizhou, 3 volumes of Yunnan, 4 volumes of Sichuan, and currently 3 volumes of Guizhou were published in the National Publishing House in July 2015. This move has completely changed the situation that the ancient Yi literatures of Yunnan, Guizhou, and Sichuan provinces did not know each other. As long as they learn the common Yi language pinyin, they can read the ancient Yi literary literatures.

Yunnan Publishing Group Company, Yunnan People's Publishing House and Yunnan Nationalities Publishing House jointly published the "Practical Encyclopedia of Rural Work" prepared by the Propaganda Department of the Yunnan Provincial Committee, which was published in a total of six volumes. 400,000 words, popularized in the local Yi area.

4) Enhance the language image of the Yi nationality

Up to now, the Red River Hani and Yi Autonomous Prefecture in Yunnan Province has exchanged the placards of more than 5000 party of government, business, and shops in the state into general Yi norms, and most of the signs in Chuxiong Yi Autonomous Prefecture have been rewritten. At the same time, a series of rules using the general Yi characters are also issued. Leaders of primary and secondary schools in other ethnic groups mainly require Yi translation of essays for school cards.

Overall, the implementation of the "General Yi Language Standard Scheme" has achieved certain results, but there are certain difficulties, especially the implementation of Guizhou Province and Guangxi Zhuang Autonomous Region is still facing a severe test, and further thinking is needed to find a suitable path of.

\section{Path Selection}

Based on the above analysis, the current path of possible choices should be based on the principle of "respecting history, learning from each other, using each other, and gradually moving closer together", establishing a holistic view of the standard of essays, based on the objective reality of the application of essays, and maintaining the study of ancient Yi texts and the stability of general essays. Treat and handle the opinions of all parties with caution. In other words, we can continue to promote the standardization of the work along the existing work, and it is not appropriate to make major changes.

However, some problems existing in the current work should also be actively dealt with, which are manifested in the following aspects: 1) The problem of variant characters can be sorted out through systematic and in-depth research, and the "Comparative Table of Variant Forms" can be compiled to adjust and accurately determine the variants. Provide a basis for Yi language education and Variant character processing; 2) Some characters of names and place names can be reserved in a limited way, but the scope of their use should be clarified; 3) The 
relationship between ancient Yi character and normative Yi character should be To clarify the difference and function of the two, it is necessary to be related to each other and to be different. 4) For homonyms or multi-words, it should be adjusted scientifically and reasonably in accordance with the use of localities and standardized in time. 5) According to the actual situation of the characters used in the four provinces and districts, and in conjunction with the development characteristics of the general norms, compile the "Comparative Tables of Yi character of Various regions", strengthen the coordination of the use of the texts in various places, and provide the basis for the promotion of the standard. 6) Focus on promoting the Yi language image from the various language areas, the language of the landscape, the language of the media, and the language used by the residents in various areas.

\section{Conclusion}

The normative work of any text has a certain historical stage. It must be based on the reality of the application of words in a certain period of time, follow the laws of language and characters, and adapt to the needs of social communication. The normative work of the text is naturally the same (Huang, 2007: pp. 2-7). From the theoretical and practical aspects of language norms, the norms of language and writing are not a one-off event. They require the joint efforts of the government, society, the public, and educational institutions to complete. We hope that under the efforts of local language experts, scholars, Yi language work institutions and other scholars who love Yi language work, the standardization work of Yi character can take a step forward, and provide valuable experience for scientific protection and development of national language and writing work.

\section{Foundation Item}

Supported by the youth research fund of humanities and social sciences of the Ministry of Education "Investigation and research on the cognition of 'General Yi Language Standard Scheme' and the current usage of word bank" (16YJC740028); Supported by the Fundamental Research Funds for the Central Universities, Southwest Minzu University "Research on the Use of Chinese Loanwords and the Characteristics of Word Order Based on the Dynamic Circulation Corpus of Yi Language" (2019NQN31).

\section{Conflicts of Interest}

The author declares no conflicts of interest regarding the publication of this paper.

\section{References}

Huang, D. K. (2007). On the Realistic Basis and Path Choice of Chinese Characters. Language and Text Applications, 2-7.

Jia, H. X., \& Sha, M. L. Y. (2014). Prospects for the Development and Development of 
“General Yi Language Standard Scheme”. Journal of Southwest Miinzu University (Humanities and Social Sciences), 9.

Jia, H. X., \& Sha, M. L. Y. (2015). The Practical Effect Level Achievements of "General Yi Language Standard Scheme". Journal of Southwest Minzu University (Humanities and Social Sciences Edition), 12.

Ma, J. W. (2012). On the Origin and Development of Yi Character. Beijing: National Publishing House.

Wang, Y. X. (2007). National Language Policy and National Identity. China National News.

Yang, Y. J. (2002). Summary of Ancient Yi Character in Guangxi. Proceedings of International Symposium on Ancient Literature and Traditional Medicine Development of Yi Nationality, Yunnan Nationalities Publishing House, 378-387.

Zhou, Y. G. (2017). The Tradition of Chinese Language and Culture and the Evolution of Ancient Language Policy. Language Strategy Research, 5. 